\title{
Prosumers y la profesionalización del periodismo ciudadano: El caso www.eurovision-spain.com
}

\author{
Prosumers and the professionalization of the citizen journalism: The case \\ www.eurovision-spain.com
}

\author{
Dra. Laura Cervi \\ Universidad Autónoma de Barcelona | Carrer de la Vinya, 738, 08193, Cerdanyola del Vallès, \\ Barcelona | http://orcid.org/0000-0002-0376-0609 | laura.cervi@uab.cat

\section{Dr. Carles Marín Lladó} \\ Universidad Rey Juan Carlos | Camino del Molino, s/n, 28943, Fuenlabrada, Madrid | \\ http://orcid.org/0000-0001-7456-5889 | carles.marin@urjc.es

\section{Carlos Sanandrés} \\ Universidad Autónoma de Barcelona | Carrer de la Vinya, 738, 08193, Cerdanyola del Vallès, \\ Barcelona | http://orcid.org/0000-0003-3220-7586 | carlos.sanandres@uab.cat
}

Fechas | Recepción: 28/01/2021 | Aceptación: 19/02/2021

\section{Resumen}

El avance imparable de la tecnología en la sociedad de la comunicación e información de hoy en día ha provocado que el modelo tradicional de comunicación vertical y unidireccional de los medios de comunicación de masas haya sido reemplazado por otro horizontal y multidireccional, donde los individuos se han convertido en productores y consumidores de contenido. Con internet en su máximo esplendor y con los teléfonos inteligentes como principales benefactores del "periodismo móvil", las herramientas de esta profesión se han puesto a disposición de las personas, provocando el nacimiento del

\section{Abstract}

The advancement of technology in the information society has replaced the traditional vertical and unidirectional communication model of the mass media with a horizontal and multidirectional one, where individuals have become prosumers. With the internet at its peak and with smartphones and "mobile journalism", the tools of this profession have been made available to people, causing the birth of so-called "citizen journalism", where anyone can be a creator and container of journalistic material. Traditional journalism already competes with digital journalism, and with initiatives of all kinds of journalistic 
llamado "periodismo ciudadano", donde content with thousands, and even millions, of cualquier persona puede ser creador y followers who consume a certain specific contenedor de material periodístico. $Y$ tanto es product. In this study, the role of "citizen así, que el periodismo tradicional ya compite no solo con el digital más elaborado o con el más modesto, sino con iniciativas de todo tipo de contenido periodístico que viene avalado más por la ilusión que por la profesionalidad, pero que, en cualquier caso, cuenta con miles, e incluso millones, de seguidores que consumen un producto determinado $y$, a la vez, específico. En este estudio, se analiza el papel del "periodismo ciudadano" a través de la web informativa dedicada al Festival de Eurovisión www.eurovision-spain.com. La finalidad es saber si esta iniciativa, impulsada por fans, acaba profesionalizando su actividad y si su eficacia abre el comienzo de una nueva era de democratización de la información o, por el contrario, incide en el final de la profesión del periodismo, tal y como se ha escenificado en los últimos cincuenta años.

Palabras clave: periodismo ciudadano, Keywords: citizen journalism, prosumer, web, prosumer, web, Eurovisión.

journalism" is analyzed through the informative website dedicated to the Eurovision Song Contest www.eurovision-spain.com

The purpose is to discover if this initiative, driven by fans, ends up professionalizing its activity and if its effectiveness opens the beginning of a new era of democratization of information or, on the contrary, affects the end of the profession of journalism, as it has been staged in the last fifty years.

Eurovision.

\section{INTRODUCCIÓN}

Cada gran invento tecnológico como la imprenta, la radio y la televisión ha supuesto un cambio de paradigma comunicativo; sin embargo, pocos momentos de la historia de la humanidad han sido tan revolucionarios, en términos de distribución de la información y del conocimiento, como el periodo actual, con la irrupción de internet a finales del siglo XX y su consolidación en la primera década del XXI (Pérez Tornero y Varis, 2012).

En los últimos 150 años hemos tenido en esencia dos clases de comunicación: a) la que va de un interlocutor a muchos participantes (libros, periódicos, radio, televisión) y b) la que se dirige de interlocutor a interlocutor (cartas, telegramas y teléfonos). Internet ha irrumpido como una gran fuerza transformadora y ha creado un nuevo paradigma comunicativo de potencialidades y posibilidades infinitas (de un interlocutor a muchos interlocutores, de muchos a uno, de uno a uno, de muchos a muchos, de humanos a objetos, de objetos a humanos, etc.) que ha marcado la transición de la sociedad industrial a la sociedad digital o del conocimiento (Santoveña-Casal, 2018).

El modelo de comunicación vertical y unidireccional, típico de la sociedad industrial y de los medios de comunicación de masas, está siendo (ha sido) reemplazado por uno horizontal y 
multidireccional (Aparici y García-Marín, 2018), en el que los individuos son productores y, a la vez, consumidores de contenido, así como emisores y receptores. Es lo que Axel Bruns (2008) denominó como "produsage", haciendo referencia a la naturaleza dual del ciudadano digital que se convierte en prosumer (prosumidor), ya que consume y produce información a la vez como un actor comunicativo más. Son materiales e informaciones que se comparten de manera física y digital en lo que el semiótico J.P. Gee (2005) define como "affinity spaces", espacios físicos o de conexión de redes en los que las personas acceden y comparten unos conocimientos previos y unos intereses comunes con la voluntad de intercambiar o aprender sobre un objeto de estudio determinado.

La llegada y el asentamiento definitivo de las nuevas tecnologías han propiciado un cambio en la correlación de fuerzas de los tradicionales agentes y mercados de la información, de la comunicación y de la cultura (Marín, 2017). De hecho, para el filósofo italiano Gianni Vattimo (2010) la postmodernidad se define propiamente como una especie de 'torre de Babel informativa', donde la comunicación y los medios adquieren un carácter central de tal calibre que las mismas ideas de postmodernidad y de pensamiento débil están estrechamente relacionadas con el desarrollo del escenario multimedia.

La red, y su consiguiente telaraña generadora de enlaces, permite a la gente conectarse con la información y con otras personas, formando de esta manera un nuevo concepto de coordinación social (Segura Vázquez, 2015).

Según Zizi Papacharissi (2010), nos encontramos ante una "ciudadanía líquida" de personas que, interactuando entre ellas, son capaces de reconstruir "el mapa de las periferias cívicas, políticas y sociales", produciendo principalmente una forma propia de periodismo, es decir, a partir de este cambio de paradigma -que implica la democratización de la informaciónasistimos inevitablemente a la democratización del periodismo.

Desde la llegada de la internet, las herramientas del periodismo han cambiado. Con relativamente poca inversión, un ciudadano común dispone (casi) del mismo material que años atrás sólo podía tener el profesional de la mesa de su lugar de trabajo: la redacción. En concreto, el mismo teléfono inteligente (Smartphone) que cualquier persona lleva en el bolsillo es un equipo multifuncional y versátil que incluye cámara para grabar vídeo y hacer fotos; que funciona como registrador de audio y herramienta de edición; y que puede realizar Live Streaming, subir contenido en tiempo real a la red y que permite, al mismo tiempo, interactuar con la audiencia.

Esos smartphones poseen un extraordinario poder a la hora de capturar hechos con una inmediatez que los medios tradicionales no se pueden permitir (Allan, 2017); por esto, los (hasta ahora) potenciales usuarios de un medio pueden convertirse en reporteros desde el mismo lugar y tiempo en el que acontece un hecho y, además, incorporarlo a los flujos de información de manera inmediata (Lydersen, 2020).

El auge de esta forma de "periodismo móvil", entendido como periodismo ejercitado con el uso de dispositivos portátiles (Cervi, Pérez y Tejedor, 2020), se ha hecho especialmente evidente en los atentados más recientes (Barcelona, Londres y París) o en las graves catástrofes naturales de la última década como el terremoto de Haití de 2010, los incendios de Australia de finales de 2019, o la actual pandemia del coronavirus. 


\section{DEFINICIÓN DE PERIODISMO CIUDADANO}

Este cambio de paradigma abre la puerta al periodismo ciudadano, un fenómeno que describe un mare magnum de prácticas difíciles de encasillar (Ferrari, 2017). Y tanto es así que hasta el momento no existe ninguna definición satisfactoria, ni siquiera una única nomenclatura definitiva de este fenómeno (Allan, 2013). Aun con todo, el "periodismo ciudadano" suele ser la fórmula más usada, si bien se habla indistintamente de periodismo ciudadano, periodismo participativo, periodismo público o periodismo democrático para referirse a este concepto. Aun así, la elección de una u otra definición parece depender más del foco de atención que de las diversas y diferentes prácticas profesionales que incluye esta forma de hacer periodismo tan de moda hoy en día (Bejarano, Limones y Mosquera, 2020).

\subsection{Los optimistas}

Muchos discursos académicos tienden a ser optimistas en la confección de lo que podría o debería ser el periodismo ciudadano, llegando incluso a considerar que esta revolución tecnológica superará el rígido modelo jerárquico de los medios de comunicación (Deuze, 2009) y nos conducirá a una "conversación abierta" (Bruns, 2008), en la que los periodistas renunciarían a su monopolio de productores de noticias y se convertirían en los nuevos "conductores del debate público" (Burum, 2016), hechos convertidos en noticias que ya no serían una historia "objetiva", sino una narrativa heterogénea construida a partir de múltiples voces, y eso, en última instancia, conduciría a una reforma sustancial de la calidad de nuestras democracias en un sentido participativo.

Estos enfoques tienden a referirse a una interpretación, a menudo idealizada, del concepto de inteligencia colectiva propuesta por Aggarwal, Malone y Woolley (2009), para hacer referencia a esa actividad de colaboración basada en un modelo de comunicación móvil, omnipresente y asíncrono, en el que se confunde lo público y lo privado. Esta definición está directamente vinculada a la idea de smart mob, multitud inteligente, de Howard Rheingold, entendida como el grupo de personas que "pueden colaborar incluso si no se conocen entre sí, gracias al uso de dispositivos con capacidad de comunicación y cálculo" (2002: 88). Este grupo de gente, en contra de la connotación negativa habitual de "masa", se comporta de manera inteligente o eficiente debido al crecimiento exponencial de las conexiones en la red.

\subsubsection{Los alternativos}

Otro enfoque vinculado a los conceptos antes mencionados ve al periodismo ciudadano como una respuesta a la incapacidad o culpa -de los medios de comunicación principales- de proporcionar información veraz. En otras palabras, según estos autores, si el "cuarto poder" se ha alejado de los ciudadanos -perdiendo credibilidad-, la ciudadanía, a través de la tecnología, asumiría el rol de perro guardián, de depositario de los medios, en el caso de que estos últimos sirvieran intereses distintos a los de la propia ciudadanía.

Es por ello que muchas personas concentran la definición de esta nueva forma de hacer "periodismo" casi exclusivamente en el lado "opcional" o "revolucionario"; por ejemplo, según Radsch (2016), el "periodismo ciudadano" es una forma alternativa y activista de elaboración de noticias que opera fuera de las instituciones y los medios tradicionales, a menudo en respuesta a deficiencias en el campo del periodismo profesional que utilizan prácticas periodísticas similares, pero que están impulsados por objetivos e ideales diferentes y 
dependen de fuentes alternativas de legitimidad, en comparación con el periodismo tradicional o convencional. Carpentier (2016), por otro lado, sostiene que es necesario centrarse en "decisiones sobre el uso de tecnologías de producción, sobre el uso del espacio y los lugares, de la visibilidad de los participantes, del uso del lenguaje, los géneros y los formatos, etc."

En el contexto iberoamericano, especialmente en América Latina, este enfoque está conectado con la idea de comunicación comunitaria y la emancipación de las propias comunidades. En este sentido, Clemencia Rodríguez (2001) define el periodismo comunitario como un concepto que implica una interacción colectiva "que cuestiona los códigos sociales, las identidades legítimas y las relaciones sociales institucionalizadas como un medio para empoderar a la comunidad".

\subsubsection{Los críticos}

Por otro lado, encontramos a los críticos hiperpesimistas, quienes consideran que estas prácticas son una forma de ejercicio amateur de la profesión y, por lo tanto, centran sus tesis en señalar los peligros potenciales que crearían tanto en el periodismo como en toda la sociedad. De hecho, según el exdirector de "Le Monde diplomatique", Ignacio Ramonet (2011) estamos observando la transformación "desde los medios de comunicación de masas hacia una gran masa de medios", con grandes oportunidades, pero también con muchos riesgos para la circulación mundial de información.

En este sentido, numerosos académicos y comentaristas de todo el mundo han destacado la multiplicación exponencial de distorsiones multimedia (con respecto tanto a los criterios de investigación de la verdad como a los de utilidad), la sobreabundancia de información que oculta y ahoga las noticias más importantes, la primacía de la cantidad (de clics, seguidores y

"me gusta") sobre la calidad de los contenidos y la supremacía de la necesidad de velocidad sobre la de profundizar que, en lugar de aumentar las posibilidades de aprendizaje, elimina las exigentes formas de pensamiento, de reflexión y concentración, e implica una renuncia sustancial a la información en profundidad (Maistrello, 2010).

Por este motivo, varios autores, entre ellos Vincent Maher (2010), han decretado la muerte del periodismo ciudadano, y muchas asociaciones profesionales, como la Federación de Asociaciones de Periodistas de España (FAPE), tienden a considerarlo como algo que "no es ni periodismo ni ciudadano" (2016).

En el mismo lado de la barricada nos encontramos con muchos intelectuales marxistas y postmarxistas que, a pesar de ser igualmente críticos, lo son por razones diametralmente opuestas a lo explicado, interpretando la cultura amateur "libre" de internet, la llamada "economía de regalo para el intercambio de información" (...) "como una especie de hechizo que, en realidad, ocultaría una lógica de movilización del trabajo inmaterial "útil e instrumental para la explotación capitalista" (Hardt y Negri, 2000:166).

Sin entrar en el debate, en este trabajo usaremos una definición más neutra, limitándonos a considerar el periodismo ciudadano como el desarrollo de la actividad periodística propiamente dicha por parte de los civiles (Cervi, 2017), si bien queremos remarcar que no son profesionales titulados ni preparados para ejercer funciones periodísticas. Por otro lado, Jay Rosen (1999) afirma que "cuando las personas, antes conocidas como audiencia, emplean las 
herramientas de prensa que tienen en su poder para informarse mutuamente están haciendo periodismo ciudadano". Shayne Bowman y Chris Willis (2003), también lo consideran como el acto de un ciudadano, o un grupo de ciudadanos, con un rol activo en el proceso de recogida, análisis y difusión de noticias e información.

De hecho, a pesar de las diferencias definitorias propias de la literatura especializada, todos los autores acordarían que las diversas formas de periodismo ciudadano comparten las siguientes características:

- Publicación abierta a partir de herramientas de bajo coste y fácil uso.

- Edición colaborativa.

- Distribución directa de contenidos a través de plataformas de interacción, como blogs o redes sociales.

Estas características son aplicables precisamente a diferentes ámbitos de la vida y son las que, según S.C. Lewis, K. Kaufhold, y D.L. Lasorsa (2010), han llevado al éxito esta práctica en un momento en el que, debido a la multitud de fuentes, se cuestionan los canales oficiales y la necesidad constante de nueva información y material que provoca, en consecuencia, un consumo rápido y casi constante.

\section{OBJETIVO Y METODOLOGÍA}

El objetivo de este artículo es analizar el papel del periodismo ciudadano en el contexto de la información relacionada con el Festival de Eurovisión y el público hispanohablante. Se trata de estudiar el caso de la web, creada por fans, más longeva del sector: www.eurovisionspain.com, que actualmente recibe una media de 6.000 visitas diarias, cifra que se puede duplicar en mayo, mes en el que se celebra el evento televisado de grandes audiencias sobre el que gira dicha web. Según datos publicados por el propio sitio en el año 2015, la web vivió "seis récords consecutivos de audiencia los días 17, 19, 20, 22, 23 y 24 de mayo, este último con 214.903 accesos y 71.250 visitantes, lo que mantiene a eurovision-spain.com como la web más leída en castellano en la temática eurovisiva y, por primera vez, nos convierten en la página sobre el festival con más audiencia mundial, solo por detrás de los medios oficiales".

\section{EUROVISIÓN, CONCEPTO DE PROGRAMA DE TELEVISIÓN CON AUDIENCIAS MILLONARIAS}

El Festival de la Canción de Eurovisión es un concurso televisivo de carácter anual, nacido en 1956, que enfrenta a cantantes y grupos musicales de diferentes países, en su mayoría europeos. En la actualidad, el ganador del certamen es el que logra una mayor puntuación, obtenida por jurados profesionales y por el voto popular de cada país participante.

Pero los orígenes debemos encontrarlos en la Europa de posguerra de los años 50 del siglo pasado y en su intento por reconstruirse. Una de las acciones podemos hallarla en la Unión Europea de Radiodifusión (UER) y su puesta en marcha del aludido festival de la canción internacional inspirado en el "Festival di San Remo" italiano. La idea fue reunir a cantantes que representaran las televisiones de los países miembros y retransmitir simultáneamente la competición musical para todos integrantes de la UER.

Además de una finalidad cultural y de hermanamiento europeo, el Festival fue percibido como un experimento tecnológico televisivo en directo, en una época en que una retransmisión de 
este tipo, sin televisión por satélite, se consideraba como algo exigente en su ejecución y, además, de prestigio.

Los países que acceden al concurso de televisión deben ser miembros activos de la Unión Europea de Radiodifusión. Pueden ser países no europeos o que pertenezcan al Consejo de Europa, si previamente han solicitado su ingreso en la UER y cumplen los requisitos para formar parte como miembros activos. Así pues, participar en eurovisión no viene determinado por el hecho de estar dentro de Europa y no tiene que ver, necesariamente, con la Unión Europea.

La ciudad de Lugano, en Suiza, acogió la primera edición del Festival de Eurovisión el 24 de mayo de 1956. Es considerado el espacio de televisión más longevo que se transmite en el mundo y el más importante en telespectadores: entre 100 y 600 millones, si se suman todos los países que participan; en 2019, por ejemplo, hubo 41 países que presentaron sus canciones y se retransmitió para todo el mundo a través de televisiones y canales de internet como Youtube.

Eurovisión se financia por parte de las televisiones que lo emiten, la mayoría públicas, además de patrocinadores, llegándose a convertir en un momento único incluso para fomentar el turismo del país y la ciudad-sede que alberga el concurso.

La final del Festival de Eurovisión se emite desde 1963 el sábado de una noche de mayo actualmente a las 21 horas (hora española)-, pero desde 2008 se programan dos galas previas eliminatorias (el martes y el jueves anterior) debido al gran número de países participantes, lo que provoca una seria competición entre naciones para llegar a la final donde solo compiten los mejores clasificados, a excepción del Big Five. Este grupo de cinco países está formado por Alemania, Francia, Italia, Reino Unido y España, que son los que más dinero aportan a la Unión Europea de Radiodifusión y, por ello, se aseguran pasar directamente a la final del concurso.

En los últimos años, el espectáculo televisivo ha dado lugar a una gran contienda entre países para presentar no solo a sus mejores intérpretes y actuaciones, sino también para mostrar una puesta en escena vistosa que llame la atención y lograr, posteriormente, el mayor número de votos. Para ello, cada país dispone de ensayos previos que permiten perfeccionar la puesta en escena, la iluminación de cada actuación y, por supuesto, una realización que haga disfrutar a los telespectadores desde sus casas. Todo este proceso hace que el auténtico fan eurovisivo lo disfrute durante toda una semana, puesto que la prensa hace seguimiento durante días y, además, en los últimos años han surgido todo tipo de portales digitales y canales en internet profesionales o no-, que se disponen a contentar a los millones de fans que tiene Eurovisión a lo largo del mundo. $Y$ todo ello ha provocado el nacimiento y consolidación, como veremos más adelante, de webs específicas, como la del presente estudio, que se muestran como auténticos hacedores del llamado periodismo ciudadano.

\section{EL ORIGEN DEL FANDOM EUROVISIVO}

Durante sus casi seis décadas de emisión, la Gala Final de Eurovisión se ha convertido en el programa no deportivo más seguido de la televisión. La última edición alcanzó los 186 millones 
de telespectadores en más de 40 países $^{1}$. En los últimos años la popularidad de este concurso musical está en máximos históricos, pero no siempre ha sido así. A finales de los años 70 perdió respaldo de audiencia y comenzó un declive que se mantendría hasta finales de los 90, cuando se introdujeron nuevas maneras de enganchar a la audiencia, más pasiva en aquellos momentos. Desde entonces hasta hoy también se creó el primer club de fans oficial del show televisivo. La "Organización General de Amantes de Eurovisión" (OGAE) se fundó en la localidad de Savonlinna (Finlandia) en 1984. Su presidente fue Jari-Pekka Koikkalainen y ya en aquel momento definió su club como no gubernamental, apolítico y sin ánimo de lucro.

Diversos estudios de comunicación diferencian un fan de un espectador corriente por la "intensidad de su relación emocional e intelectual" con el producto que está consumiendo (Jenkins 2012: 56). Así pues, es habitual que tiendan a organizarse, como apunta Fiske (1992), para cohesionarse ante la interpretación dominante que suele despreciar el programa, la serie o la canción por carecer de prestigio cultural. Por esta razón, no es de extrañar que el primer club de fans nazca en Finlandia, un país que había entrado en la competición el mismo año que España (1961) y que hasta 1984 su mejor resultado había sido un sexto puesto con Marion Rung y la canción "Tom, Tom, Tom".

El éxito de esa Organización fue tan grande que en unos meses comenzó su expansión internacional. En 1987 nació en España, concretamente en Madrid, el primer club de fans de Eurovisión, que se coordinó con la OGAE Internacional. Hoy en día existen 40 delegaciones, 39 de las cuales corresponden a participantes de Eurovisión y una más, creada en 2005, que aglutina a los seguidores cuyos países no concursan en el Festival o que no han solicitado su ingreso en la UER.

En el caso de España, un grupo de fans del festival decidió crear, en 1989, una organización paralela a la OGAE. Se fundó así, la "Asociación de Eurovisivos de España" (AEV-España). Hoy por hoy, la $A E V$ es la única asociación de estas características en el ámbito español inscrita legalmente en el Registro Nacional de Asociaciones, de acuerdo con la Ley Orgánica 1/2002 que regula el Derecho de Asociación. Su funcionamiento es similar a la OGAE, en cuanto a la publicación de fanzines y concursos entre sus inscritos.

Según datos facilitados por la propia organización, la AEV está compuesta por aproximadamente 200 personas y son los fans quienes se encargan de redactar y actualizar la web, que se ha convertido en la decana en cuanto a noticias sobre Eurovisión en España. La asociación también fue pionera en el ámbito de medios en papel del certamen musical. En 1990 lanzaron "Eurovisivos", revista que posteriormente se llamaría "Olevisión" y que tendría su propia web de contenidos: www.olevision.com

Sin embargo, a partir de la llegada de la banda ancha en España, el user-generated content (UGC) digital, es decir, los contenidos creados por los usuarios -o fans para el caso que nos ocupa- serán una constante en el universo eurovisivo, que se nutre de estos materiales durante todo el año. En este flujo latente de información encontramos a www.eurovisionspain.com, la web fan con mayor número de visitantes de España e Hispanoamérica, y que es

\footnotetext{
${ }^{1}$ En 2020 no hubo Eurovisión por la pandemia mundial del coronavirus. Las cifras son de 2019. Recuperado el 17 de enero de 2021 en http://www.eurovision-spain.com/el-big-5-suma-mas-de-lamitad-de-la-audiencia-de-eurovision-2019/
} 
objeto de este estudio. Aunque su volumen de información no es regular, en los meses previos al evento aumenta de forma notable, si bien 24 horas después de la final de Eurovisión decae por el lógico desinterés, una vez alcanzado el clímax y la finalización del programa televisado. Entre los materiales más habituales encontramos reseñas web y videorankings elaborados a través de sus propias predicciones, aunque la muestra puede ser de lo más variada, como vídeos con propuestas de puestas en escenas o videojuegos que reproducen el festival. A medida que los usuarios han adoptado más habilidades digitales, la complejidad y el contenido se han ido profesionalizando, llegando a ser incluso más innovadores que los ofrecidos por el canal digital oficial: www.eurovision.tv, y por el canal de emisión encargado de retransmitirse en España: www.rtve.es/eurovision

Con la llegada de las redes sociales, durante la segunda mitad de la década de los 2000, las comunidades fans se expandieron. Ya no sólo fue a través de la web o de las plataformas de vídeo, sino que trasladaron su pasión a los nuevos medios, creando grupos en redes como Facebook o sus propias aplicaciones. Con la inmediatez de las redes sociales, éstas se convirtieron en un factor clave y los fans agradecieron este cambio de paradigma al que se sumaron de manera natural. Con el tiempo y la tecnología, ha sido mucho más fácil e inmediato publicar un tuit o un estado de Facebook que un artículo en una web o un vídeo. Tanto la AEV como la OGAE-Spain se han ido adaptando a estos cambios, pero el ganador de la batalla ha sido la segunda organización a través de su plataforma www.eurovision-spain.com. A continuación, detallamos la cronología.

\section{LA PROFESIONALIZACIÓN DE WWW.EUROVISION-SPAIN.COM}

En mayo del 2000 se lanzó eurofestival.net, una web muy simple con información y fotografías de Eurovisión. Por aquel entonces, el ADSL empezaba a llegar a los hogares españoles y aquel sitio estático no permitía interacción entre los navegantes. Después de cinco meses y ante el interés generado por la comunidad fan, el maestro de educación primaria, Óscar Abilleira, y un equipo de seguidores del certamen decidieron darle un empuje y convertirlo en algo más ambicioso. El 15 de diciembre de 2000 nacía el prolegómeno de www.eurovision-spain.com, una idea que se forjó con el nombre de www.esctoday.spain.com, siguiendo el modelo de otros países. Fue el empresario ejecutivo y escritor neerlandés, Sietse Bakker, fundador y editor de esctoday.com y, años después, de la web oficial del certamen www.eurovision.tv, quien muestra el camino al equipo de fans españoles para crear esctoday.spain.com, versión española de la web www.esctoday.com. El equipo español recibió valiosos consejos, directrices y soporte técnico para la creación de ese portal. 




Figura 1: El portal de noticias www.esctoday.spain en junio del 2001

Fuente: http://eurovision-spain.com/

Fueron meses de trabajo hasta llegar, en septiembre de 2001, a una versión 1.3 con lenguaje php y un sistema para que los colaboradores pudieran insertar de forma autónoma noticias. A finales de ese mismo año, con un nuevo formato ya 2.0, aparecieron novedades como los sistemas de votación y una pestaña dedicada al talent musical de TVE para elegir al representante de Eurovisión 2002, "Operación Triunfo", que arrasó en audiencias en España. Todo ello obligó al fichaje de personal para la web y a personajes como McCobo, Mashiel y Juana Interpovincial.

Los resultados no se hicieron esperar: aumento del tráfico y de la publicidad de la web. Uno de los factores fue, sin duda, el hecho de que fuera un sitio gratis y no de pago. Solo un año más tarde desde su nacimiento, se profesionalizó de forma definitiva con el dominio vigente hoy en día: www.eurovision-spain.com: 


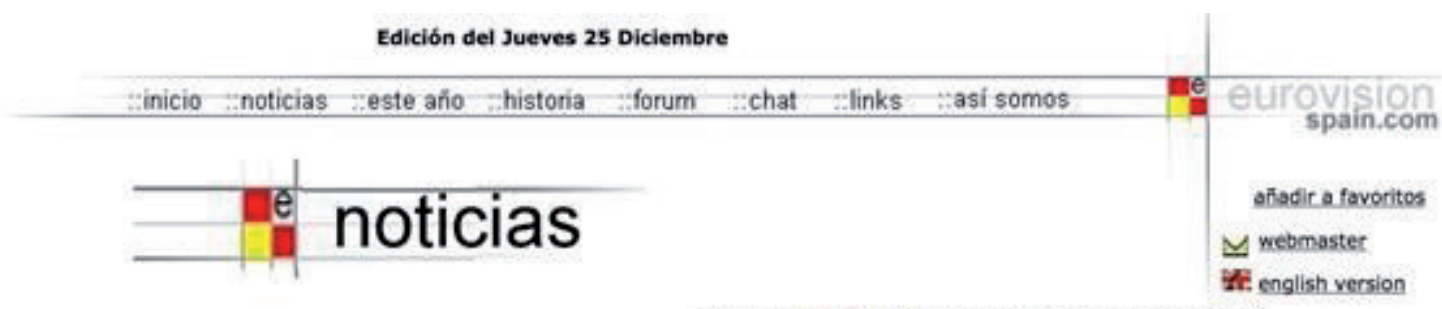

it Faltan $\mathbf{- 4 5 9 8}$ dias para Eurovision (25-Mayo-2,002)

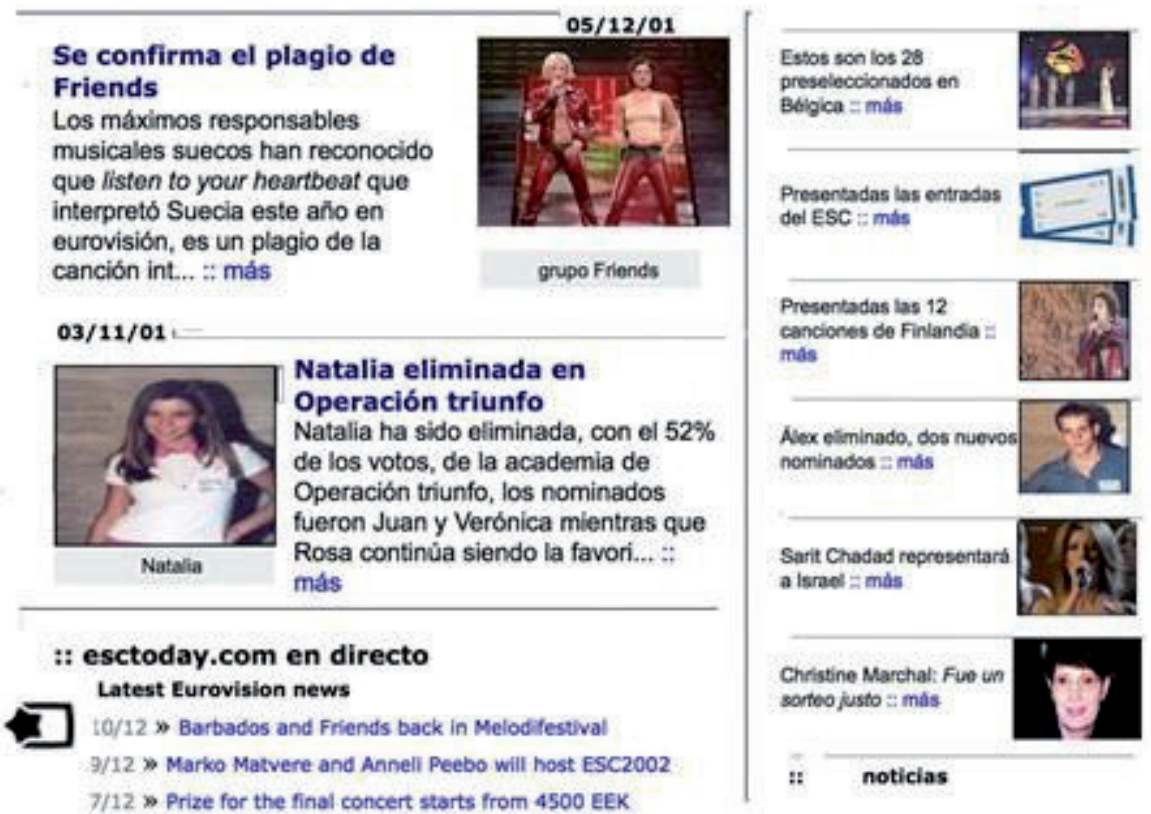

Figura 2: Primera versión del portal www.eurovision-spain.com en mayo del 2002

Fuente: http://eurovision-spain.com/

Tras el éxito de "Operación triunfo" y una vez finalizado el festival en mayo de 2002, la web logró acumular hasta 15.000 visitas diarias durante la semana anterior a la final del concurso. El 1 de junio de 2002 apareció un diseño más moderno de la web y nuevos contenidos con la información más explícita y detallada: reportajes, corresponsales, chats, buscador de contenidos históricos de Eurovisión, juegos, tonos y melodías para móvil, etc.

Pero la web objeto de este estudio no dejó de crecer en todos los sentidos. El 19 de octubre de 2002, y ante el incesante número de nuevos visitantes, se abrió a noticias en otros idiomas como el sueco, italiano o francés, y también a las tres lenguas cooficiales del Estado español: catalán, euskera y gallego. Desde el portal se realizó un seguimiento de Eurovisión 2003 por parte de los corresponsales de la web en Riga (Letonia), ciudad donde se llevó a cabo ese año el concurso, y por primera vez www.eurovision-spain.com se acreditó como medio para acceder al Festival, gracias a las credenciales que consiguieron los fans acérrimos que también estaban detrás de la gestión del sitio web. 


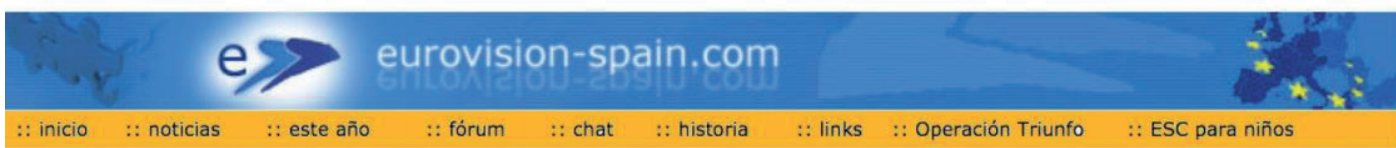

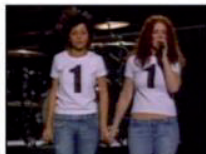

\section{$06 / 04 / 03$ \\ BRAVO: Las Tatu \\ irán a eurovisión}

Parece que el boom del año pasado cuando todos los medios de comunicacion se hacían eco de Festival de Eurovision apoyando a Rosa y a nuestra canción, parece que este año con Beth, las revistas y prensa de nuestro país están siguiendo día a día las noticias que les llegan desde cualquier punto de Europa...."

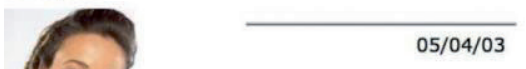

TELEINDISCRETA: Europa al ritmo de Beth

La revista TELEINDISCRETA, publica esta semana en portada, un reportaje sobre nuestra representante en Eurovision, Beth...."

7 : noticias de esctoday.com

04/08 » Emily Reed to OGAE Second Chance Contest 4/08 " The Frank Naef show is back in Israel 04/09 " No kissing t.A.T.u. on Turkish television $04 / 08$ " Turkey favourite among Norwegian fans $04 / 07$ " Riga will have an intensive cultural week
$04 / 09$ » Norwegian Television informs about I atvis

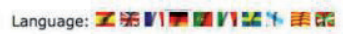

05/04/03 ::La canción de Tatu será interpretada en ruso $"$

05/04/03 ::Turquía emitirá los videos del ESC 2003 a partir del lunes "

03/04/03 ::La canción de Tatú ya está disponible en la red "'

01/04/03 ::Programación eurovisiva del Canal Nostalgia de TVE

31/03/03 ::Presentada la canción de Bélgica "

28/03/03 ::Rusia-2003: ¿en ruso o en inglés?

27/03/03 ::El videoclip de Dime se presentará el 1 de abril $\mapsto$

:: más noticias $\gg$

Faltan 44 dias 8 h. 23 m.

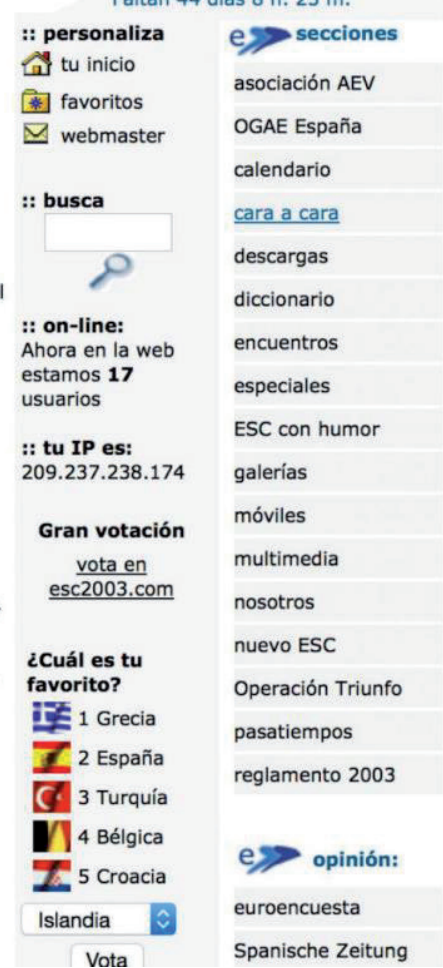

Figura 3: El portal www.eurovision-spain.com en el año 2004

Fuente: http://eurovision-spain.com/

En la segunda mitad del 2003, ante el crecimiento imparable de la web, el fundador de www.eurovision-spain.com abandonó esctoday.com para dedicarse en cuerpo y alma a la web sobre Eurovisión con más éxito y audiencia. Se genera así un portal wap con interfaz de diario digital y, sobre todo, con un foro propio que propiciará más movimiento e interacciones entre visitantes-fans del sitio, convirtiéndose en la web sobre Eurovisión más visitada de toda España, sobre todo después del cierre de www.eurofestival.net.

Con el final de Eurovisión 2004, y tras superar el millón de visitantes en julio de ese mismo año, www.eurovision-spain.com inició una reestructuración con un rediseño, nuevos colaboradores y secciones. 


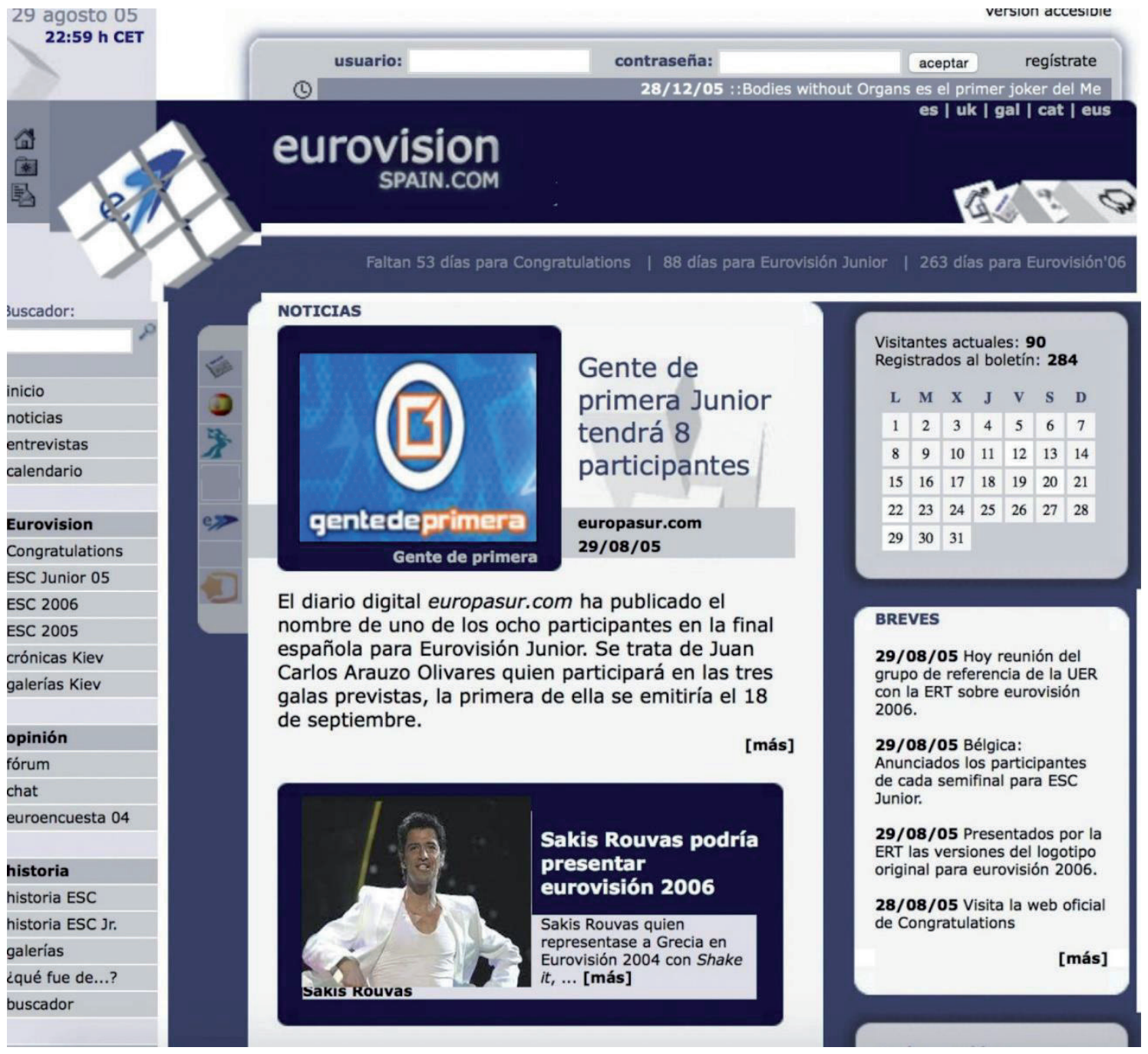

Figura 4: El portal www.eurovision-spain.com en el año 2004

Fuente: http://eurovision-spain.com/

En junio de 2005 se dio un paso más en el sitio web, permitiendo al usuario personalizarla y creando nuevas secciones como un calendario de eventos y una galería multimedia donde se colgaron las primeras fotografías de la sede del Festival de ese año, adelantándose de esta manera a los medios periodísticos tradicionales en papel, pero también a los digitales y a los de corte audiovisual.

Con la nueva versión 7 de www.eurovision-spain.com presentada en julio de 2006, el trabajo se intensificó con la incorporación de vídeos, así como de comentarios que debían ser autorizados previamente; se lograba con esta acción evitar injurias, calumnias y conflictos entre usuarios.

Miguel Loredo Garay se convirtió en el nuevo propietario de la web el 1 de agosto de 2007 y, con él, el 15 de diciembre de ese año se estrenó la versión 8.0, que llega a los 5 millones de visitas, según datos aportados por el portal objeto de análisis. La interactividad de la web y la mejora de todo lo relacionado con aspectos multimedia se convierten así en las principales novedades, además de perfeccionar lo existente hasta ese momento. 


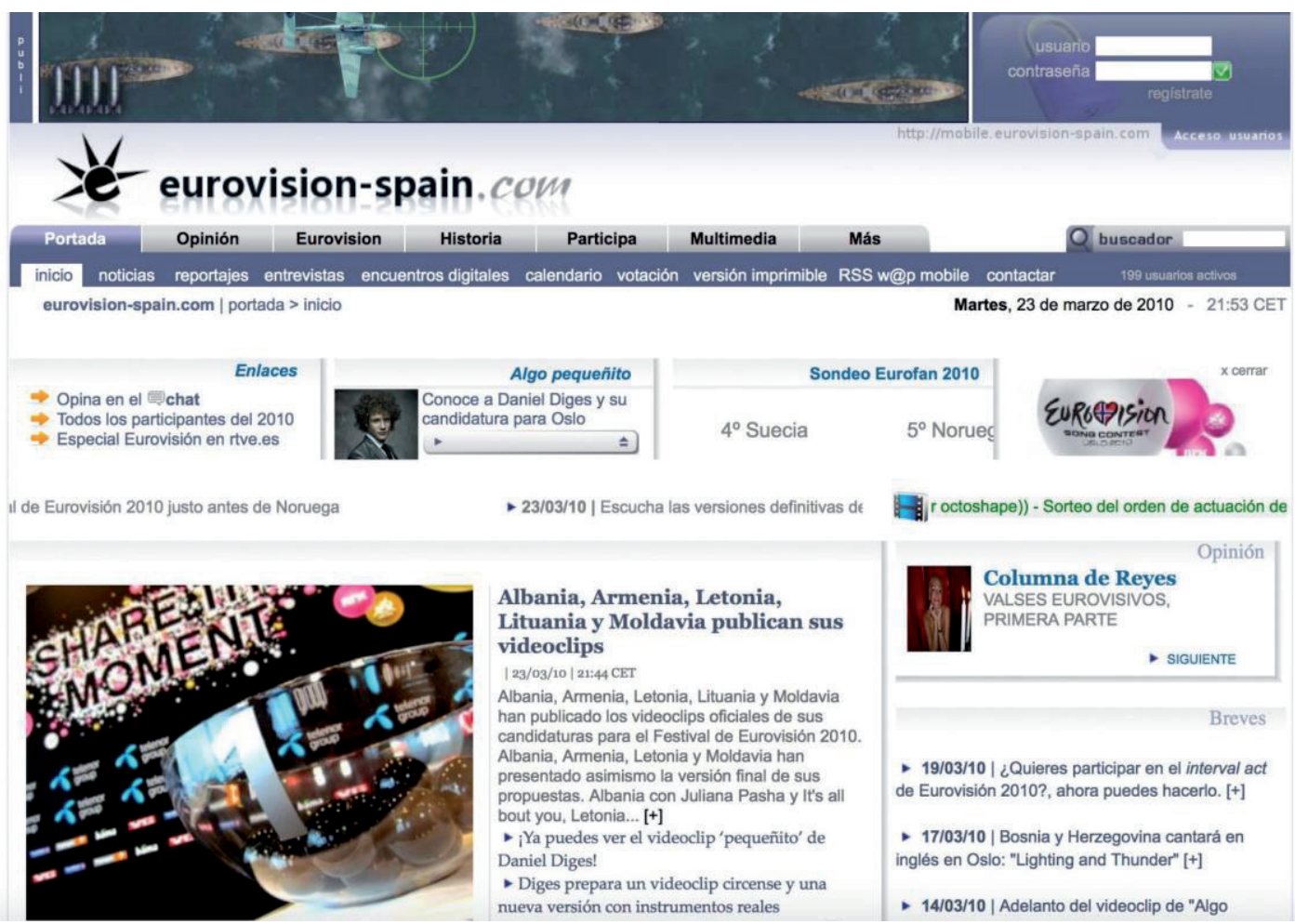

Figura 5: El portal www.eurovision-spain.com en año 2010

Fuente: http://eurovision-spain.com/

Entre 2013 y 2014 la web siguió aumentando su calidad de almacenaje para soportar el incremento de visitantes que llegaron hasta los 80.000 durante la semana en que se emitieron las semifinales y la final del Festival.

Con la campaña web: \#eurometamorfosis se introdujo, a principios de 2015, un diseño y funciones que han llegado hasta la actualidad. Además, fueron pioneros en la filosofía del responsive web design.

El 14 de marzo de 2016, José García Hernández (Luka) se hizo cargo de la dirección de www.eurovision-spain.com y, meses más tarde, nace el Club E-S de www.eurovisionspain.com, con servicios preferentes para los socios, convirtiéndose así en referencia que traspasa el universo digital. Además del respeto de los seguidores de Eurovisión, esta web logró especializarse definitivamente en la temática eurovisiva y su creatividad propició que sus fans/gestores -no profesionales del periodismo- se convirtieran en columnistas de referencia no solo para el sector eurofan, sino para los periodistas que durante años han cubierto el festival. 


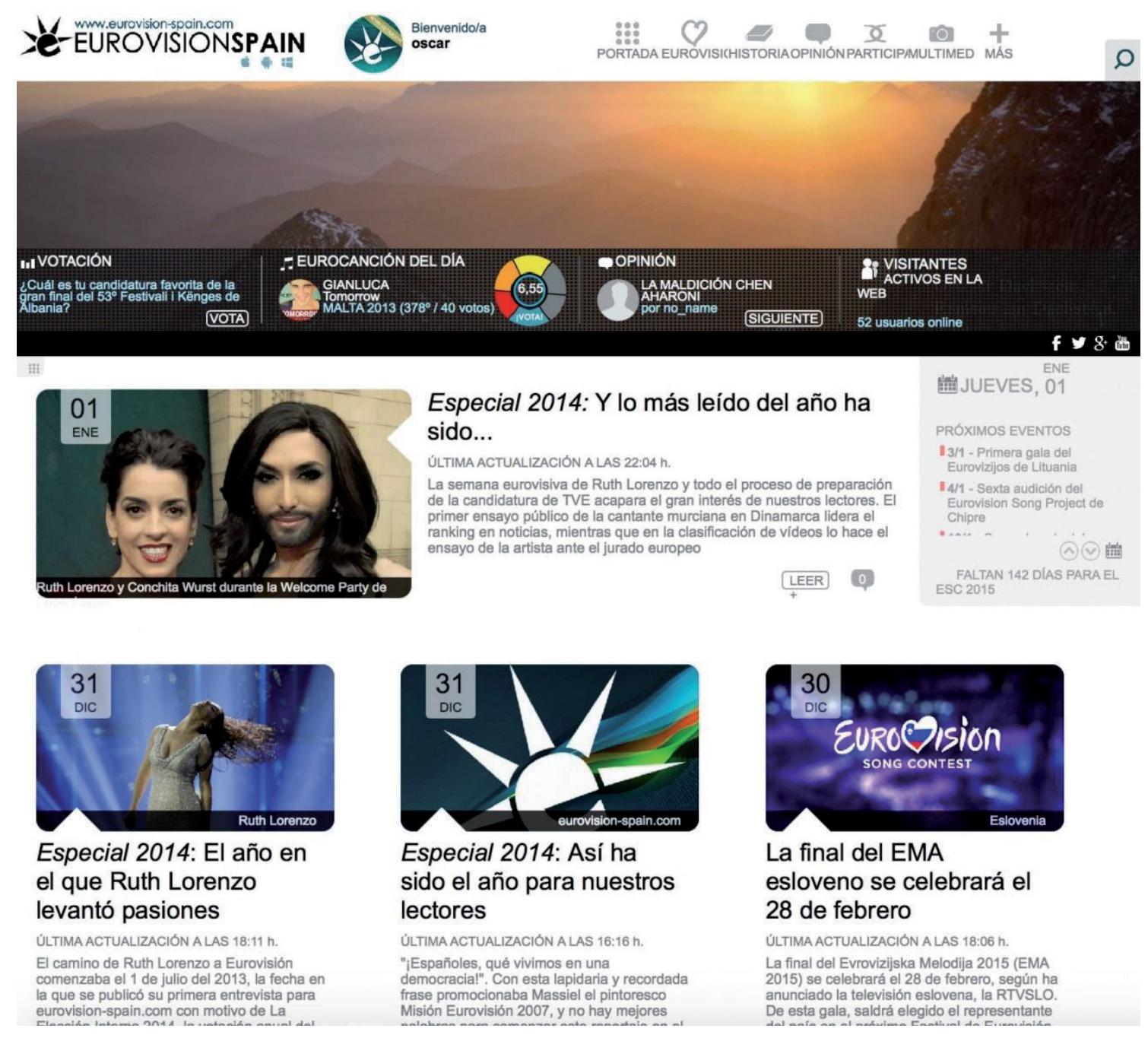

Figura 6: El portal www.eurovision-spain.com en 2014 tras una remodelación que duró un año Fuente: http://eurovision-spain.com/

\section{DISCUSIÓN Y CONCLUSIONES}

El análisis descriptivo que hemos realizado de la web www.eurovision-spain.com es un claro ejemplo de un portal de noticias de referencia, creado a partir del periodismo ciudadano, que se erige como servicio periodístico de un evento musical de audiencias millonarias, y retransmitido por televisión, que genera interés social y audiencia digital, además de la tradicional audiovisual. En este caso, es una iniciativa impulsada, desde el principio, por fans que han acabado profesionalizando su actividad sin ser profesionales de la información. El periodismo ciudadano, por lo tanto, puede no ser el fin del periodismo; al contrario, podría ser el comienzo de una nueva era que da mucha esperanza a la democratización de la información y al perfeccionamiento de la democracia. Otro debate sería, y queremos insistir en ello, que este tipo de "periodismo" sea intrusismo y por qué se escenifica como tal.

Sea como sea, puede que el periodismo ciudadano acabe dominando el qué (agenda) y el cuándo (inmediatez), pero los periodistas profesionales siempre serán necesarios para añadir el cómo y el por qué (contextualización). 
Sin embargo, la profesión periodística debe reinventarse y pasar de una estructura jerarquizada, donde el periodista era el generador y seleccionador único de las noticias, a otra donde el periodista se comunica con un público organizado en comunidades virtuales y con un rol activo en la construcción de la noticia (Cervi, 2019). El periodista debe convertirse, pues, en el moderador de un gran debate. Un debate cada vez más abierto y que tiene lugar en un nuevo ecosistema informativo más amplio, rico y variado. En este sentido, tal y como afirmaba Dan Gillmor (2004), es cierto que "nuestros lectores, colectivamente, saben más que nosotros"; entonces, uniendo la formación, la experiencia y las habilidades del periodista profesional con la inteligencia colectiva y la capacidad de aportar de sus lectores, se puede generar un periodismo colaborativo y deliberativo capaz de curar la salud de una esfera pública cada vez más debilitada y, por ende, regenerar nuestras democracias.

Finalmente, hoy más que nunca, queda patente que la condición sine qua non para que se produzca una colaboración fructífera y de calidad es la existencia de una educación en medios, al alcance de todos y continua a lo largo de la vida, que ayude tanto a los ciudadanos como a los propios profesionales a desarrollar las capacidades críticas y las habilidades necesarias para poder aprovechar todas las potencialidades que las tecnologías ofrecen.

\section{Referencias}

Aggarwal, I., Malone, T.W. \& Woolley, A. W. (2015). Collective Intelligence and Group Performance. Current Directions in Psychological Science, 24 (6), 420-424.

Allan, S. (2017). Photojournalism and Citizen Journalism: Co-operation, Collaboration and Connectivity. Routledge.

Allan, S. (2013). Citizen Witnessing. Cambridge, MA, Polity.

Aparici, R. \& García-Marín, D. (2018). Comunicar y educar en el mundo que viene. Gedisa.

Bejarano, Z., Limones, V. \& Mosquera, F. (2020): Redes sociales y periodismo ciudadano: investigación documental. Journal of science and research, (1), 149-164. https://doi.org/10.5281/zenodo.3599276

Bowman, S.; Willis, C. (2003). We Media | How audiences are shaping the future of news and information. Reston VA. The Media Center at The American Press Institute. Disponible en https://bit.ly/3eim9rh

Bruns, A. (2008). Blogs, Wikipedia, Second Life, and Beyond: From Production to Produsage, Peter Lang.

Burum, I. (2016). Democratizing Journalism through Mobile Media: The Mojo Revolution. Routledge.

Carpentier, N. (2016). Beyond the Ladder of Participation: An Analytical Toolkit for the Critical Analysis of Participatory Media Processes. Javnost - The Public, 23(1), 70-88.

Cervi, L.; Pérez Tornero, J.M.; Tejedor, S. (2020). The Challenge of Teaching Mobile Journalism through MOOCs: A Case Study. Sustainability (12), 5307. https://doi.org/10.3390/su12135307

Cervi, L. (2019). Comunidades virtuales de viajeros: un caso de éxito. Universitas, Revista de Ciencias Sociales y Humanas, (30), 97-125. https://doi.org/10.17163/uni.n30.2019.05

Cervi, L. (2017) Citizen Journalism and User Generated Content in Mainstream Media. new dialogic form of communication, user-engagement technique or free labor exploitation? Revista de Comunicaçao Dialogica, (1), 120-141. https://doi.org/10.12957/rcd.2019.41871

Deuze, M. (2009). The Future of Citizen Journalism. En Allan, S. \& Thorsen, E., Citizen Journalism: Global Perspectives, 255-264. Peter Lang. 
Federación Internacional de Periodistas (2016). "La concentración de medios en América Latina: su impacto en el derecho a la comunicación". Oficina Regional Latinoamérica y Caribe. https://bit.ly/3bpNcyT

Ferrari, P. (2017): No tempo das telas: como a presentificação alterou o consumo de informação. Razón y palabra, 21(2_97): 406-422.

Fiske, J. (1992). The cultural economy of fandom. En Lewis, L. A., The adoring audience: Fan culture and popular media (30-49). Routledge.

Gee, J. P. (2005). Semiotic social spaces and affinity spaces: From the age of mythology to today's schools. En Barton, D. y Tustin, K., Beyond Communities of Practice: Language power and social context, (9780521836432): 214-232. http//:doi.org/ 10.1017/CBO9780511610554.012

Gilmor, D. (2004). We the Media. Sebastopol, California: O'Reilly Media.

Hardt, M. \& Negri, A. (2000). Empire. Cambridge, Massachusetts \& London. Harvard University Press.

Lewis, S. C., Kaufhold, K., \& Lasorsa, D. L. (2010). Thinking about citizen journalism: The philosophical and practical challenges of user-generated content for community newspapers. Journalism Practice, 4(2), 163-179. doi:10.1080/1461670090315691

Lydersen, A. B. (2020). MOJO! Telling the story of mobile journalism, through a mobile phone, to a mobile audience (Master's thesis).

Jenkins, H. (2012). Textual poachers: Television fans and participatory culture. Routledge.

Maher, V. (2010), Citizen Journalism is Dead. Personal blog. Disponible en https://bit.ly/3szjZ3P

Maistrello, G. (2010). Giornalismo e nuovi media. L'informazione al tempo del citizen journalism. Apogeo.

Marín, C. (2017). El Informativo de televisión. Editorial Gedisa.

Papacharissi, Z. (2010). A Private Sphere: Democracy in a Digital Age. Polity Press.

Pérez Tornero, J.M.; Varis, T. (2012). Alfabetización mediática y nuevo humanismo. UOC.

Radsch,C. (2012). Unveiling the Revolutionaries: Cyberactivism and the Role of Women in the Arab Uprisings. April 2012. The Baker Institute and the Kelly Day Endowment, Rice University. Disponible en https://bit.ly/38g95P1

Ramonet I. (2011). La explosión del periodismo. Capital Intelectual.

Rodríguez, C. (2001). Fissures in the Mediascape. An International Study of Citizens' Media. Cresskill, NJ, Hampton Press.

Rosen, J. (1999). What Are Journalists For? New Haven and London: Yale University Press.

Rheingold, H. (2002). Smart Mobs: The Next Social Revolution. Basic Books.

Santoveña-Casal, S. (Coord.). (2018). Enredados en el mundo digital. Sociedad y Redes Sociales. UNED.

Segura Vázquez, A. (2015). Digitalizar y controlar: un collage de tecnologías vigilantes. Boletín ECOS noㅡ 29. Fuhem ECOsocial. Recuperado de https://bit.ly/3bsce0u

Vattimo G. (2010). Il pensiero debole. Feltrinelli. 


\section{Semblanza de los autores}

Laura Cervi es Profesora Serra Húnter del Departamento de Periodismo y Ciencias de la Comunicación de la UAB. Doctora en Ciencia Política por la Universitá di Pavia, Italia y por la UAB. Es miembro del grupo de investigación Gabinete de Comunicación y Educación, dirigido por el catedrático Dr. José Manuel Pérez Tornero en la UAB y coordinadora académica del Máster en Gestión de la Comunicación Política y Electoral del Departamento de Periodismo y Ciencias de la Comunicación de la UAB. Ha participado en diversos proyectos internacionales y ha sido, junto al Dr. José Manuel Pérez Tornero, Investigadora Principal del proyecto europeo Y-NEX, European Youth News Exchange, (2015-2017). En el año 2014 fue investigadora visitante en la Newberry Library de Chicago (EEUU).

Carles Marín Lladó es Profesor titular de Periodismo Audiovisual en la Universidad Rey Juan Carlos (URJC) desde 2003. Se ha especializado en la información televisiva y radiofónica a partir de su análisis discursivo. Desde 2012 dirige el Máster en Reporterismo de Televisión en la misma universidad. Ha publicado una decena de libros y numerosos artículos sobre reporterismo e informativos audiovisuales, y cuenta con una larga trayectoria en TV y radio como creador de formatos, director, guionista y reportero tanto de programas informativos como de infoentretenimiento. Ha sido vicerrector y vicedecano de la URJC y directivo de la Academia de las Ciencias y las Artes de Televisión (AcademiaTV).

Carlos Sanandrés es profesor asociado laboral del Departamento de Publicidad, Relaciones Públicas y Comunicación Audiovisual de la Universidad Autónoma de Barcelona donde imparte Técnicas de Redacción y Comunicación Audiovisual de las Organizaciones. Compagina su labor docente con la carrera profesional como guionista y creador de contenidos para diferentes productoras nacionales. Es licenciado en Ciencias de la Comunicación y Máster Oficial en Investigación en Periodismo mención sociedad del conocimiento en la misma universidad. En la actualidad es, además, profesor en el BA (HONS) Fashion, Marketing and Communication del Instituto Europeo de Diseño de Barcelona. 